\title{
A Framework of Photo Acoustic Imaging for Ovarian Cancer Detection by Galvo-mirror System
}

Mahmoodian $\mathbf{N}^{*}$ and Haddadnia $\mathbf{J}$

Biomedical Engineering Department, Hakim Sabzevari University, Sabzevar 397, Iran

"Corresponding author: Mahmoodian N, Biomedical Engineering Department, Hakim Sabzevari University, Sabzevar 397, Iran, Tel: 989364471400; E-mail: naghmeh.ma56@yahoo.com

Rec date: Apr 1, 2016; Acc date: Apr 20, 2016; Pub date: Apr 30, 2016

Copyright: (c) 2016 Mahmoodian N, et al. This is an open-access article distributed under the terms of the Creative Commons Attribution License, which permits unrestricted use, distribution, and reproduction in any medium, provided the original author and source are credited.

\begin{abstract}
Ovarian cancer is one of the most common types of cancer among women, which often leads to death. As a result, developing new techniques for diagnosing this disease at early stages, preventing the high rate of death in women with high-risk, and minimizing the large side effects on the economy must be considered seriously by experts. For early diagnosis of this disease, it is necessary to utilize ultrasound integrated with photo acoustic technique in which the minimal amount of cost, energy, and laser are acquired. In addition, the procedure should provide sufficient pulse to make ovaries tissue able to take image. This new clinical diagnosis technique utilizes modest pulse energy and low electrical voltage so that, it could be assumed as a proper alternative for conventional ultrasound in showing the image of abdominal ovary tissue. The researcher aims at presenting a real time photo acoustic-imaging probe for imaging human ovaries tissue; thus, this study seeks to investigate the effect of low electrical voltage and modest laser pulse energy while providing enough pulse for ovarian tissue as well as implementation issue. This model utilizes dual modality imaging approach and describes several researches carried out in order to establish dual-model imaging consisting ultrasound and photo acoustic. The system can accomplish near-simultaneous ultrasound and photo acoustic imaging. The model is capable of reaching thorough diagnostic information based on the tissue where there is only individual imaging model with its limitations.
\end{abstract}

Keywords: Photo acoustic; Ultrasound; Ovarian cancer; Laser pulse

\section{Introduction}

Photo acoustic imaging known as opto-acoustic or thermo-acoustic imaging, is able to image human and animal organs equipped with high contrast and high spatial resolution simultaneously [1].

Ovarian cancer is the fifth most common cancer among women, which often leads to death. This disease causes more death than any other type of diseases related to women's reproductive system. Despite the prevalence of the disease among women and its definite importance, it mostly cannot be diagnosed in early stages due to lack of effective screening techniques [2]; therefore, the need for new instruments and tools for the early detection of the disease is quite essential. One of the best imaging modality, being known in this area, is Photo acoustic. This technique has demonstrated great potential for imaging ovarian tissues $[1,3]$.

Photo acoustic imaging has been employed in several medical areas in higher absorption contrast. For instance, Photo acoustic microscopy is one of the useful systems, designed for imaging superficial organs with a high image quality. The second use of Photo acoustic imaging is diagnosing the presence of breast cancer in early stages. The third one is making higher resolution, compared with optical imaging in animal tissue and giving useful biomedical information about them [3]. As provided below, the imaging resolution and penetration depth of optical imaging are explained in the Figure 1 below.

For living tissues, the methods at the left of the graph are primarily limited by light scattering, whereas the methods to the right are primarily limited by light attenuation in tissue, a parameter that depends on both absorption and scattering, or by ultrasound attenuation.

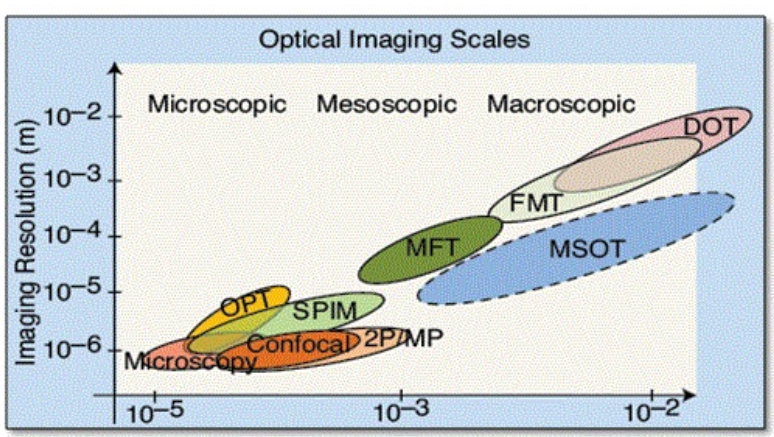

Figure 1: The penetration depth and resolution of modern photonic imaging techniques [3].

Note that optical projection tomography (OPT) and selective plane illumination microscopy (SPIM) can operate deeper than the range shown in naturally transparent or chemically cleared samples. (2P/MP: two-photon/ multi-photon microscopy; DOT: diffuse optical tomography; FMT: fluorescence molecular tomography; MFT: mesoscopic fluorescence tomography; MSOT: Multispectral optoacoustic (photo-acoustic) tomography)(Opto-acoustic Imaging Lab, Department of Biomedical Photonics, Institute of Applied Physics, University of Bern). 
Since the ultrasound can prepare mechanical properties of biomedical ovarian tissue, it is not useful in identifying cancer in early stages. Many women may suffer from cancer that is not identified yet or may undergo unnecessary oophorectomy, which is the surgical removal of an ovary or ovaries. This surgery usually happens due to diseases like cancer or ovarian cysts, in order to decrease the probability of any development in breast or ovarian cancers. Oophorectomy can even increase the survival possibility in women with high risks.

Although ultrasound method seems to provide higher resolution than optical imaging method, small tumors are not mostly divulged, applying this technique. Another reason that makes us unable to apply this method lonely in identifying cancer is that, it is not capable of visualizing blood vessels in its early stages [3]. However, employing the combination of ultrasound and photo acoustic could make us able to have both mechanical resolution and optical absorption contrast together as a Photo acoustic image. In other words, Photo acoustic imaging could be employed for identifying cancer at early stages $[1,4]$.

\section{Literature Review}

Photo-acoustic imaging (PAI) is an emerging biomedical imaging technique in which a short-pulsed laser beam penetrates diffusely into a tissue sample [1] (Potential Role of Co-registered Photo-acoustic and ultrasound). Aguirre et al. develops co-registered 3-D ultrasound and photo acoustic imaging system a 1280 -element $(10 \times 128)$ and $1.75-\mathrm{D}$ acoustic array. They achieved 3D images in range of scanning $80 \mathrm{dig}$ in azimuth and 20 dig in elevation. The system has been applied to image normal porcine ovarian tissue for ex vivo use. However, this system faces two limitations: one is about the beam steering angel, and the other is about the elevation resolution [1].

Wang et al. also presented 3D imaging system for in vivo use based on a $2 \mathrm{D}$ array ultrasound probe. A wavelength-tunable dye laser pumped by a Q-switched Nd:YAG laser serves as the light source and a modified commercial ultrasound imaging system (iU22, Philips Healthcare) with a 2D array transducer (X7-2, Philips Healthcare) detects both the pulse-echo ultrasound and photo-acoustic signals. The imaging system enables rendering of co-registered 3D ultrasound and photo-acoustic images without mechanical scanning. The resolution along the azimuth, elevation, and axial direction are measured to be $0.66 \mathrm{~mm}, 0.91$ and $0.84 \mathrm{~mm}$ for photo-acoustic imaging. These results highlight the clinical potential of 3D PAI for identification of sentinel lymph nodes for cancer staging in humans. However, they employ large amount of laser to provide enough laser energy for the system [4].

They use Photo-acoustic microscopy system to characterize of human ovarian tissue. They also extracted eight different features and higher blood vessel resolution in compared with conventional Photoacoustic images. They also classified ovarian into malignant and benign using generalized linear mode [5].

They use Photo-acoustic image to show light absorption in the ovary related to the vasculature distribution. The research also demonstrated high amount of light absorption is belong to malignant ovaries. At the end, they proved that Photo-acoustic image is promising model for diagnosis ovarian cancer [6].

Alqasemi et al research is based on FPGA parallel processing in a co-registered ultra sound and Photo-acoustic image system. They used 128-chanel to acquisition with FPGA for real time imaging of human ovaries up to 15 frames per second. The system is able to detect ovaries' vasculature by in vivo co-registered Photo-acoustic system while it is limited by the laser pulse repetition frequency of $15 \mathrm{HZ}$ [7].

In the Figure 2 below, the historical development of photo-acoustic imaging is provided. (Biomedical Photonic Imaging Group, Bio Physical Engineering Group, Faculty of Science and Technology, University of Twente, Enschede, The Netherlands).

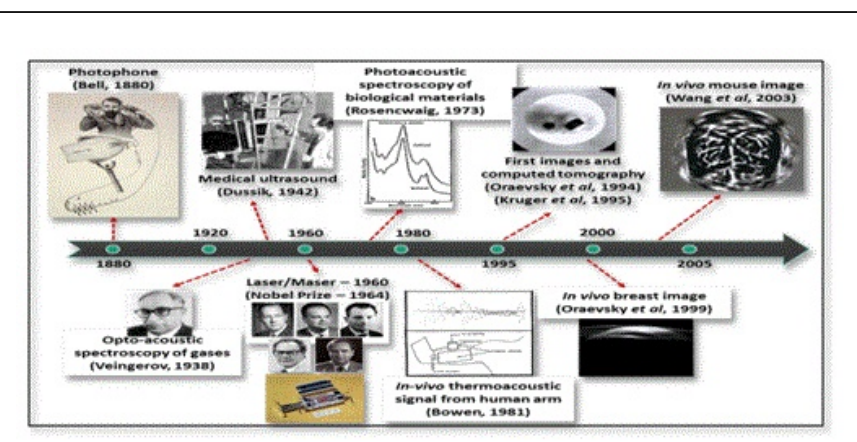

Figure 2: The historical development of photo-acoustic imaging.

\section{Preliminaries}

\section{Ultrasound imaging}

Ultrasound is one of the imaging methods employed by many people all over the world to identify a mass of tissue in the ovarian, breast, kidney, liver, prostate, pancreatic and uterine [8].

Ultrasound wave's frequency is between $20 \mathrm{KHZ}$ and $2 \mathrm{MHZ}$, while the range of human hearing (normal adult) is between $20 \mathrm{HZ}$ to 20KHZ. Therefore, Ultrasound has a greater frequency than human limitation hearing. Diagnostic ultrasound tool usually utilizes the range of $2 \mathrm{MHZ}$ to $20 \mathrm{MHZ}$ that is able to probe body of patients' tissue to construct diagnostic image with small beams [9]. Ultrasound is an oscillating sound pressure wave with a frequency greater than the upper limit of the human hearing range [9] (Figure 3).

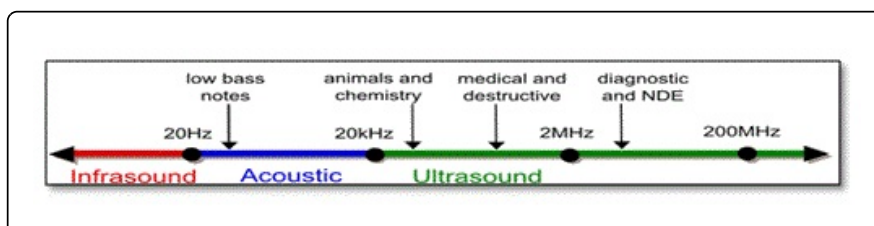

Figure 3:Approximate frequency ranges corresponding to ultrasound, with rough guide of some applications [9].

Ultrasound-based diagnostic medical imaging technique are used to visualize muscles, tendons, and many internal organs, to capture their size, structure and any pathological lesions with real time tomographic images [10] (Figure 4). 
Page 3 of 5

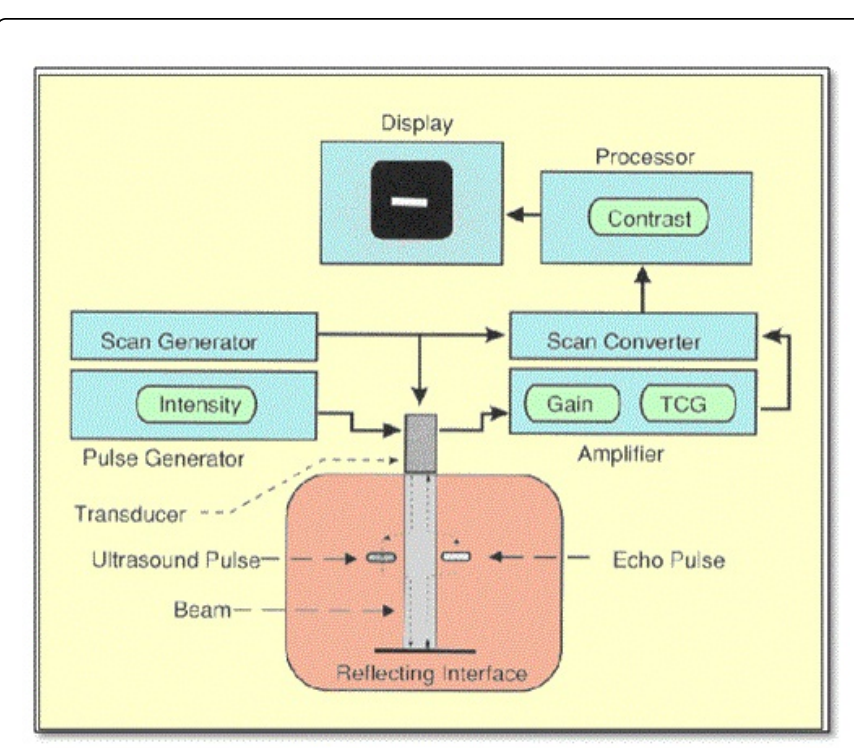

Figure 4: The principal functional components of an ultrasound imaging system [10].

The above image shows functions run by all parts of an ultrasound system, which contains computer and electronic circuits. In this system, the only part that has contact with human body is transducer. There are two functions done by transducer: 1) making ultrasound pulse and 2) receiving ultrasound echoes. There is some electricity resulting from pressure known as piezoelectric inside the transducer that performing two functions. First, by sending electrical pulse, piezoelectric begins to vibrate and produce the ultrasound and in the next step the piezoelectric produces an electric pulse, when it is vibrated by returning echo pulse from human body [9].

\section{Photo-acoustic imaging}

Photo acoustic imaging is developed based on non-ionizing laser pulse and makes a hybrid biomedical imaging modality. The process of making image is started by irradiating laser pulse energy into the biological tissue and will be absorbed by tissue and converted into heat energy. Moreover, heat energy leads to thermo-elastic expansion in biological tissue that is the cause of ultrasound emission. Then ultrasonic transducer detects the generation ultrasonic waves.

Finally, the image is formed and this formation process is known as a hybrid biomedical imaging technology. The technique is capable of showing the contrasts existing in microwave, RF and light absorption between healthy and tumor tissue with the high-resolution ultrasound. Because of the two kinds of energy, (sending leaser energy and receiving ultrasonic wave produced by body tissue) utilized in photoacoustic system, it is called hybrid technique. As the mass of blood vessels near the tumor is more than healthy tissue, it absorbs more energy. Therefore, there is more heat and finally more expansion in tumor tissue in compare with healthy tissue around it [11]. Photoacoustic tomography and photo-acoustic microscopy are two type of Photo-acoustic image $[8,12,13]$. The steps toward photoacoustic imaging of the targeted area are illustrated in Figure 5.

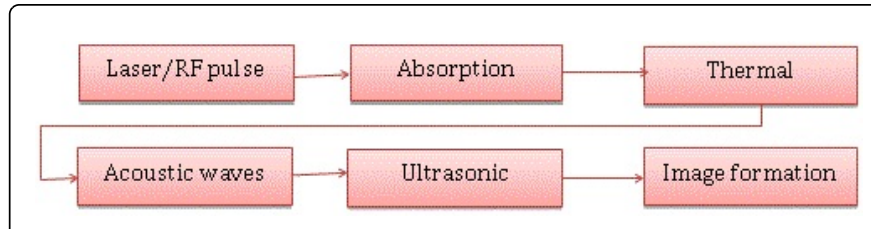

Figure 5: Schematic illustration of photoacoustic imaging.

\section{Optic}

- Non-ionized and safe radiated medical applications are provided through optical photos.

- Biochemical information is provided by optical spectra based on the absorption level, fluorescence, and Raman scattering, since molecular conformation is involved in this case.

- Angiogenesis, a kind of optical absorption which focuses on the amount of hemoglobin, and hyper metabolisms, as a symbol which shows the level of oxygen saturation in hemoglobin, are specific features of cancer.

Scattering media or turbid media is a process which points to biological tissues scattered optically. Besides, a range of $400-1350 \mathrm{~nm}$ is reported for the optical absorption in this case which is considered a weak one. The mean free path between photon scattering events and the mean absorption length are $0.1 \mathrm{~mm}$ and $10-100 \mathrm{~mm}$ successively [14]. Figure 6 shows Frequency spectrum of electromagnetic radiation imaging technologies.

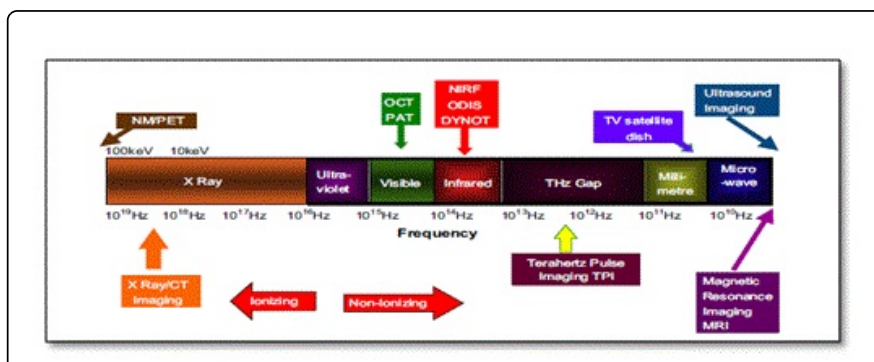

Figure 6: Frequency spectrum of electromagnetic radiation imaging technologies [15].

\section{Ovarian cancer}

Ovarian cancer occurs in ovary [1]. Cancer happens because of abnormal cells entering and spreading in the body. Unfortunately, there is no symptom in early stages. There are only bloating, abdominal swelling, and pelvic pain in later stages. Therefore, the women who are at high risk of this type of cancer are recommended to remove their ovary and sometimes because of false positive tests, they undergo an unneeded surgery. The only way to show correct diagnosis is to do biopsy and send the sample tissue to lab for microscopic analyses.

\section{The stage of ovary cancer based on Wikipedia indicated in follow:}

Stage I - Including one of the ovaries or both of them: 
- IA - Regarding just one of the ovaries, consists of a capsule intact no tumor was observed on ovarian; including negative washings

- IB - Both ovaries are involved here; a capsule intact and no tumor was; negative washings

- IC - If there would be a capsule ruptured, a tumor, or positive washings on ovaries, a tumor would be limited to ovaries

\section{Stage II - Stretching the pelvis:}

- IIA - Enlarging the uterus or fallopian tube; declaring negative washings

- IIB - Reforming the pelvis and its structures; negative washings are displayed

- IIC - Revising the pelvis and its structures along with positive peritoneal washings

Stage III:

- One or both of the ovaries or even the fallopian tubes are suffered from the cancer; moreover, following events might occur;

- The disease maybe extended beyond the pelvis to the lining of the abdomen

- The disease may be extended to lymph nodes in the back of the abdomen, called retroperitoneal lymph nodes;

Stage IV (any T, any N, M1): This stage is considered as a leadingedge, since the spleen, liver, lungs, or even other organs located outside the peritoneal cavity, are suffering from cancer. (The peritoneal cavity is the area surrounded by peritoneum, which lines the inner abdomen and some of the pelvis and mostly masks its organs).

Therefore, to overcome the problem of high mortality because of no adequate imaging technique, new imaging techniques need to be provided to diagnose this disease at early stages. Photo-acoustic imaging emerged as a technique that can show image of neovascularization associated with tumor angiogenesis with laser pulse and then combine it with ultrasound image. The ultrasound transducer is used to reconstruct images of photo-acoustic waves produced by short-pulse laser beam to sample tissue and make thermo-elastic expansion around the tissue [1].

\section{Methodology Applied for This Purpose}

\section{In developing a framework}

The process of ovarian cancer diagnosis in Photo-acoustic system is started from laser irradiation (1064 nm laser). The source of irradiation is a portable fiber laser, which provides $1064 \mathrm{~nm}$ laser. This is not a bulky laser, which is produced by Q-switch.in the current laser system in ovarian cancer detection.

The laser will reach to galvo-mirror, which moves the laser beam by rotating the mirror. A function generator is needed to produce continuous scanning applications. The galvo-mirror produces a uniform illumination beam into biological tissue.

The area which the galvo-mirror covered by producing the uniform illumination beam into biological tissue is approximately $3 \mathrm{~cm}$ in diameter. In the cases where the ovary was bigger than the illumination beam, the images were acquired at different locations to cover most of it. This is non-ionizing process and tends to observing energy by tissues. In this step, the energy that is observed by tissue will be converted into heat. According to a theoretical principle, heating leads to thermo-elastic expansion. Then, the phenomenon leads to ultrasonic emission.

The ultrasound system, which operates under two modes (Photoacoustic mode and pulse-echo mode) by ultrasonic transducer, detects both ultrasonic waves and Photo-acoustic waves. Consequently, both Photo-acoustic and ultrasonic images are reconstructed, based on array of transducer. The accuracy of the model is determined, compared with the conventional ultrasound.

\section{Formulation of the framework}

Ovarian cancer has the highest mortality of gynecologic and there is no adequate technique to detect it at early stages; thus, there is an urgent need for finding effective methods for early cancer detection.

New imaging techniques provide high contrasts for ovarian cancer, based on observing energy by tissue, such as Photo-acoustic imaging. The laser is the most important part of this system and new technology of using modest laser pulse energy makes it ideal for real-time imaging.

In this work, galvo-mirror technique is applied to send lower pulse energy, producing ultrasound emission, then the transducer receives Photo-acoustic and ultrasound waves to reconstruct the image that is useful for cancer detection in early stages.

As cancer detection in methodology, after laser triggers the main purpose of the framework discussion as follow Figure 2.

- The first section (S1) is called laser irradiation containing trigger pulse to ultrasound, and Photoacoustic. The pulse-echo in both ultrasound and Photoacoustic is the vibration. The vibrations are passed through the tissue. So the pulse moves away from the source, low electrical voltage (diode laser pump) instead of highvoltage. The field programmable gate array (FPGA) module provides a trigger pulse to ultrasound, PA and galvo mirror. It also synchronizes the ultrasound and Photoacoustic operation.

- The second (S2) is called galvo mirror action containing FPGA and function generator. The FPGA synchronizes the operation of ultrasound, Photoacoustic and galvo mirror. The function generator controlees the galvo mirror movement, and the galvo mirror moves the laser beam by rotating which is caused by continues scanning of function generator. The galvo-mirror produces a uniform illumination beam into biological tissue.

- The third step (S3) is known as laser scanning. After the laser is incident on the mirror, new cycle of mirror begin. Consequently, ultrasound pulse echo occur and the events of alternating ultrasound and PA repeat. The numbers of ultrasound pulse echo are 6 times more than PA pulse.

- As a result, the image reconstruction (S4) provides the image of the ultrasound in the gray scale mode and the image of the PA in colorful mode. Yet, noises in ultrasound images reduce contrast and limiting distinguish abnormal region, very good contrast can be given by PAI through tissue optical absorption. The ultrasound system having the ability of receiving ultrasound and PA signals. Array transducer of ultrasound also reconstructs the ultrasound and PAI.

\section{The Proposed Procedure}

Using photo acoustic system to detect ovary cancer through galvomirror was developed in previous framework. The system consists of ultrasound imaging system (commercial type) for detecting ultrasound 
Page 5 of 5

and PA signals, fiber laser which is the source of beam irradiation with radius of $0.5-\mathrm{mm}$ incident on scanning mirror with angle relative to ultrasound propagation, galvo-mirror system moving the laser beam by rotating the mirror, and function generator to control the galvomirror system movement, FPGA for providing trigger pulse for all of ultrasound, laser beam, and function generator. Figure 7 shows the parts of imaging system. The system is suitable for ex vivo imaging of ovaries.

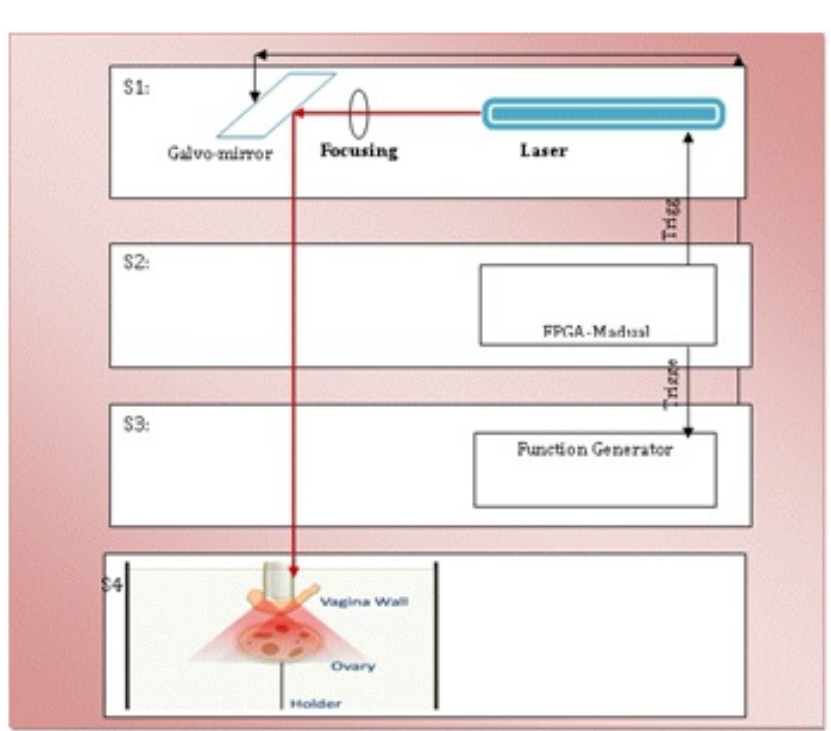

Figure 7: The parts of imaging system.

\section{Conclusion}

According to the researcher's experiments, photo acoustic imaging system is detected by low voltage and laser beam and is proposed by other type of imaging system.

In order to detect cancer images in ovaries, the proposed framework should be considered as follows:

In the S1 part of Figure 7, displays the preparation of irradiation of laser by modest pulse energy and low electrical voltage. In addition, pulse trigger is provided to ultrasound, $\mathrm{PA}$ and galvo mirror

The S2 part depicts the application of galvo-mirror to move laser beam to the desirable tissue by rotating. Function generator controls the rotation of galvo-mirror.

Totally, 112-laser and PA pulse-echo beams complete each cycle of mirror which is shown in Figure 7.

At the end part, i.e., S4 of Figure 7 construction of image by ultrasound system is provided. Following the protocol, a very good PA image can be obtained.
Therefore, the benefit of the framework is detecting ovaries cancer by the low voltage and modest laser beam. In addition, the framework was proposed to show PA as a clinically system which can be used easily. Then use it with a logical price.

In this study, all part of the system presented and the PA image constructed by modest laser beam. As a future work of this study, the model could be implemented as a PA system for real time non-invasive evaluation of ovaries for detecting ovary cancer in early stages. Moreover, the model would increase the women's health who are exposed to ovarian cancer.

\section{References}

1. Aguirre A, Guo P, Gamelin J, Yan S, Sanders MM, et al. (2014) Coregistered three-dimensional ultrasound and photoacoustic imaging system for ovarian tissue characterization. J Biomed Opt 14.

2. Brewer MA, Utzinger U, Barton JK, Hoying JB, Kirkpatrick ND, et al. (2004) Imaging of the Ovary. Technol Cancer Res Treat 3.

3. Xu M, Wang LV (2006) Photoacoustic imaging in biomedicine. Rev Scilnstrum 77.

4. Wang Y, Erpelding TN, Jankovic L, Guo Z, Robert JL, et al. (2012) In vivo three-dimensional photoacoustic imaging based on a clinical matrix array ultrasound probe. J Biomed Opt 17.

5. Wang T, Yang Y, Alqasemi U, Kumavor PD, Brewer M, et al.(2013) Photoacoustic microscopy for ovarian tissue characterization. Proc SPIE 8581.

6. Aguirre A, Ardeshirpour Y, Sanders MM, Brewer M, Zhu Q (2011) Potential role of coregisteredphotoacoustic and ultrasound imaging in ovarian cancer detection and characterization. TranslOncol 4: 29-37.

7. Alqasemi U, Li H, Yuan G, Kumavor P, Zanganeh S, et al. (2013) Realtime interlaced ultrasound and photoacoustic system for in vivo ovarian tissue imaging. Proc SPIE 8581.

8. Fass L (2008) Imaging and cancer: A review. MolOncol 2: 115-152.

9. Sprawls P, Ultrasound Production and Interactions.

10. Corso JF (1963) Bone-Conduction Thresholds for Sonic and Ultrasonic Frequencies. J AcoustSoc Am 35: 1738.

11. Joines WT, Zhang Y, Li C (1994) The measured electrical properties of normal and malignant human tissues from 50 to $900 \mathrm{MHz}$. Med Phys 21: 547-550.

12. Oraevsky AA, Avateeva EV, Solomatin SV, Karabutov AA,Valeri GA, et al. (2002) Optoacoustic imaging of blood for visualization and diagnostics of breast cancer. Proce SPIE 4618: 81-94.

13. Pogue BW, Poplack SP, McBride TO, Wells W, Osterman KS, et al. (2001) Quantitative hemoglobin tomography with diffuse near-infrared spectroscopy: pilot results in the breast. Radiology 218: 261-266.

14. Hsin IW, Wang LV (2007) Biomedical optics: principles and imaging. John Wiley \& Sons.

15. Wikipedia (2010) Approximate frequency ranges corresponding to ultrasound, with rough guide of some applications. Wikipedia. 